\title{
Probing the Galactic s-process nucleosynthesis using barium stars
}

\author{
Shejeelammal Jameela, Aruna Goswami \\ Indian Institute of Astrophysics, Koramangala 2nd Block, Bangalore - 560034, India
}

\begin{abstract}
The origin and evolution of neutron-capture elements in our Galaxy is poorly understood. In this work, we have attempted to understand the Galactic s-process nucleosynthesis using barium stars as probes. We have performed high resolution spectroscopic analysis for three barium stars HD 36650, HD 207585 and HD 219116. The analysis of HD 219116 is based on HCT/HESP data at a resolution of 60,000 covering a wavelength region 3530-9970 A. For HD 36650, we have used the FEROS spectrum at a resolution of 48,000 covering the wavelength region 3520-9200 Å. For HD 207585, the spectrum from VLT/UVES at a resolution of 48,000 covering the wavelength region $3290-6650 \AA$ is used. Our analysis clearly shows that the surface chemical composition of these barium stars are enriched by s-process nucleosynthesis products coming from their former companion low-mass AGB stars. A discussion on the distriburtion of abundance ratios based on the existing nucleosynthesis theories is presented.
\end{abstract}

Keywords: stars: Abundance - stars: chemically peculiar - stars: nucleosynthesis

\section{Introduction}

All the elements beyond Fe-peak are produced through the slow and rapid neutron-capture processes. The Asymptotic Giant Branch (AGB) stars are the predominant sites for the slow neutron-capture (sprocess) nucleosynthesis. Low- and intermediate-mass stars evolve through the AGB phase of stellar evolution. The s-process occurs at low neutron densities of $N_{n} \approx 10^{7}-10^{10}$ neutrons $/ \mathrm{cm}^{3}$ (Busso et al. 1999) on a timescale much slower than the $\beta$-decay rate of unstable isotopes. The important neutron sources for this process are ${ }^{13} \mathrm{C}(\alpha, \mathrm{n}){ }^{16} \mathrm{O}$, for the stars with initial mass $\leq 3 M_{\odot}$, and ${ }^{22} \mathrm{Ne}(\alpha$, $\mathrm{n})^{25} \mathrm{Mg}$, for the stars with initial mass $\geq 4 M_{\odot}$. The dominant isotopes of $\mathrm{Sr}, \mathrm{Zr}, \mathrm{Nb}, \mathrm{Ba}$, La and $\mathrm{Pb}$ are produced through s-process. The isotopes such as ${ }^{86} \mathrm{Sr},{ }^{96} \mathrm{Mo},{ }^{104} \mathrm{Pd},{ }^{116} \mathrm{Sn}$ are produced only by the s-process. Besides these heavy elements, AGB stars are important contributors of carbon and nitrogen in the Galaxy. About a third of the total carbon in the Galaxy is found to be produced in AGB stars (Dray et al. 2003). Major producers of ${ }^{14} \mathrm{~N}$ in the Galaxy are the intermediate-mass AGB stars with Hot-Bottom Burning (Henry et al. 2000). The products of the nucleosynthesis from the interiors are brought to the surface of the AGB star through the Third Dredge Ups and these materials are expelled to the ISM through stellar winds. The understanding of the AGB phase and nucleosynthesis is important to study the role of low- and intermediate-mass stars in the chemical enrichment of the Galaxy.

However, observations show that the neutron-capture elements in the Galaxy show a large scatter at a given metallicity. The observed scatter in the abundances of these elements with respect to 
metallicity suggest that the ISM from which the stars are formed may not be mixed homogeneously or same element may have different origins. The analysis of Cayrel et al. (2004) shows that the elements with $\mathrm{Z}<30$ show very little scatter, discarding the claim for an inhomogeneous ISM. Detailed chemical analyses of stars with the atmosphere enriched by the neutron-capture elements can shed light on the origin and chemical evolution history of these elements in the Galaxy. Metaldeficient Barium stars constitute useful candidates for conducting such studies.

Barium (Ba II) stars belong to a family of peculiar G and $\mathrm{K}$ spectral types which were first identified by Bidelman \& Keenan (1951). They are mostly in the giant phase of stellar evolution. Their surface chemical composition exhibits overabundance of elements heavier than iron. An important characteristic property of these stars is that $\mathrm{C} / \mathrm{O}<1$ (Barbuy et al. 1992, Allen \& Barbuy 2006, Drake \& Pereira 2008, Pereira \& Drake 2009). They exhibit abnormally strong lines of Ba II at $\lambda 4554 \AA$, Sr II at $\lambda 4077 \AA$, as well as enhanced $\mathrm{CH}, \mathrm{CN}$ and $\mathrm{C}_{2}$ molecular bands. They are low radial-velocity objects and generally members of the Galactic disk. A fraction of them show mild metal deficiency. The analysis of Luck \& Bond (1991) shows that $\mathrm{C}_{2}$ molecular bands in metal-deficient Ba stars are not strong as compared to the classical Ba stars.

All giant barium stars are found to be in binaries with a now invisible white dwarf companion (McClure et al. 1980, McClure 1983, 1984, McClure \& Woodsworth 1990, Udry et al. 1998a,b, Jorissen et al. 2019). A generally accepted scenario that explains the observed high abundances of neutron-capture elements is a binary picture. These stars are believed to have received via binary mass-transfer mechanisms the nucleosynthesis products of the companion star produced during its AGB phase of evolution. Hence, the chemical composition of this class of objects can be used to trace the AGB nucleosynthesis at their corresponding metallicity.

In section 2, we presented the spectroscopic methodology. Results and discussions are presented in section 3. Conclusions are drawn in section 4.

\section{Spectroscopic Methodology}

\subsection{Object selection}

With the aim to understand the Galactic s-process nucleosynthesis using barium stars as probes, we have selected a sample of Ba star candidates from various sources (Lü 1991, Bartkevicius 1996). The results from our analysis of the three stars HD 36650, HD 207585 and HD 219116 are presented in this work.

\subsection{Data acquisition.}

The high resolution spectrum for HD 219116 is acquired using HESP attached to the Himalayan Chandra Telescope (HCT) at a resolution of $\mathrm{R} \sim 60,000$. The wavelength coverage of this spectrum spans from 3530 to $9970 \AA$. A VLT/UVES spectrum at a resolution of $\mathrm{R} \sim 48,000$ is used for HD 207585. The wavelength coverage of this spectrum is 3290-6650 $\AA$. The analysis of HD 36650 is based on a FEROS spectrum at a resolution of $\mathrm{R} \sim 48,000$, and covers the wavelength region 3520$9200 \AA$. All the spectra have $\mathrm{S} / \mathrm{N}>30$. The basic data of the program stars are given in Table 1.

\subsection{Data Reduction}

The data reduction is done using the basic tasks in Image Reduction and Analysis Facility (IRAF) software. An image frame is first trimmed to get an area that contains only useful data. The image frame is then bias subtracted followed by the flat correction. Different orders from the spectrum are 
Table 1: Basic data for the program stars.

\begin{tabular}{|l|l|l|l|l|l|}
\hline Star name & RA(2000) & DEC(2000) & Spectral type & V & Parallax(mas) \\
\hline HD 36650 (FEROS) & 052742.92 & -680427.16 & K0 III & 8.79 & $2.655 \pm 0.027$ \\
HD 207585 (UVES) & 215034.71 & -241111.68 & G2 II & 9.78 & $5.315 \pm 0.407$ \\
HD 219116 (HESP) & 231330.24 & -172208.71 & G8 III & 9.25 & $1.584 \pm 0.044$ \\
\hline
\end{tabular}

Table 2: Derived atmospheric parameters of the program stars

\begin{tabular}{|l|l|l|l|l|l|}
\hline Star name & $\begin{array}{l}T_{\text {eff }}( \pm 100) \\
(\mathrm{K})\end{array}$ & $\begin{array}{l}\log g( \pm 0.2) \\
(\text { spectroscopic) }\end{array}$ & $\begin{array}{l}\log g \\
(\text { parallax })\end{array}$ & {$[\mathrm{Fe} / \mathrm{H}]$} & $\begin{array}{l}\zeta( \pm 0.1) \\
\left(\mathrm{kms}^{-1}\right)\end{array}$ \\
\hline HD 36650 & 4880 & 2.40 & $2.78 \pm 0.01$ & $-0.02 \pm 0.13$ & 1.30 \\
HD 207585 & 5800 & 3.80 & $3.90 \pm 0.05$ & $-0.38 \pm 0.12$ & 1.00 \\
HD 219116 & 5050 & 2.50 & $2.68 \pm 0.02$ & $-0.45 \pm 0.11$ & 1.59 \\
\hline
\end{tabular}

then extracted using the task 'APALL'. The spectrum is then wavelength-calibrated using a Th-Ar arc spectrum. The wavelength calibrated image is then corrected for dispersion and continuum-fitted for further analysis.

\section{Data Analysis}

Absorption lines due to different elements are identified by comparing closely the spectra of the program stars with the Doppler-corrected spectrum of the star Arcturus. The log $g f$ and excitation potential values are taken from the Kurucz database of atomic line lists. The equivalent width of good clean spectral lines are measured using various tasks in IRAF. A master line list including all the elements was generated.

An initial model atmosphere was selected from the Kurucz grid of model atmospheres (http://cfaku5.cfa.hardvard.edu/) using the photometric temperature estimate and the log g value for giants/dwarfs. A final model atmosphere was adopted by an iterative method. The effective temperature is determined by the method of excitation equilibrium, micro-turbulent velocity by equivalent width balance and surface gravity by ionization balance of Fe I and Fe II lines.

The $\log g$ value is also calculated using the parallax taken from GAIA DR2 archive. The bolometric correction, $\mathrm{BC}$, is determined using the empirical calibration that links $\mathrm{BC}$ with the effective temperature of the star from Alonso et al. (1999). The interstellar extinction is determined as described by Chen et al. (1998). The derived atmospheric parameters of the program stars are given in Table 2.

For most of the elements, abundances are determined from the measured equivalent width of lines of the neutral and ionized atoms using the adopted model atmosphere. For the analysis, the radiative transfer code MOOG (Sneden 1973) is used with the assumption of Local Thermodynamic Equilibrium (LTE). For the elements $\mathrm{Sc}, \mathrm{V}, \mathrm{Mn}, \mathrm{Co}, \mathrm{Cu}, \mathrm{Ba}, \mathrm{La}$ and $\mathrm{Eu}$, showing hyper-fine splitting and for the elements with only single clean line in the spectra and for the molecular bands, spectrum synthesis from MOOG has been used to find the abundances. The hyper-fine components of Sc, V and $\mathrm{Mn}$ are taken from Prochaska \& McWilliam (2000), Co and Cu from Prochaska et al. (2000), Ba from McWilliam (1998), La from Jonsell et al. (2006) and Eu from Worely et al. (2013). The solar abundance values of the elements are taken from Asplund et al. (2009).

The stellar evolutionary stage of the program stars is found from their location in the Hertzsprung- 


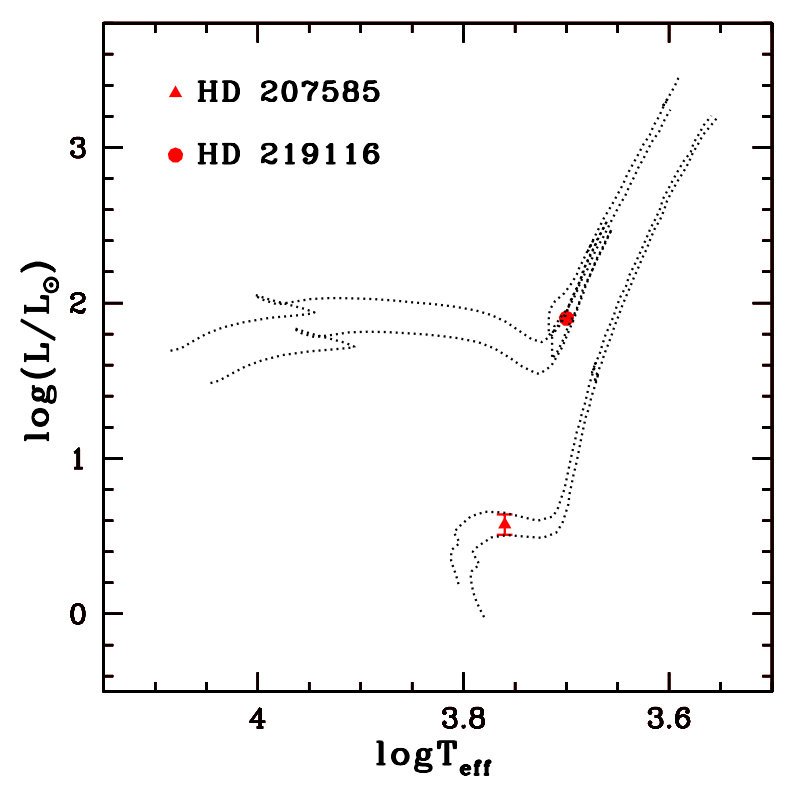

Figure 1: Location of HD 219116 and HD 207585 in the Hertzsprung-Russell diagram. The evolutionary tracks are shown for 1.0, 1.1, 2.2 and $2.5 M_{\odot}$ from bottom to top for $\mathrm{z}=0.008$.

Russell diagram (Girardi et al. 2000 data base of evolutionary tracks) using the luminosity and spectroscopic temperature estimates. The position of the program stars in the HR diagram is shown in Figures 1 and 2.

\section{Results and Discussion}

Detailed chemical abundance analysis of the program stars HD 36650, HD 207585 and HD 219116 were carried out. The absolute abundances and the abundance ratios of light elements- $\mathrm{Na}$ and $\mathrm{Mg}$ as well as neutron-capture elements such as $\mathrm{Sr}, \mathrm{Y}, \mathrm{Zr}, \mathrm{Ba}, \mathrm{La}, \mathrm{Ce}, \mathrm{Nd}$, and $\mathrm{Sm}$ are determined for these stars. The observed abundance ratios are compared with their counterparts in other barium stars from literature. The comparison is also done with the $\mathrm{CH}$ stars, CEMP stars and normal field giants from literature. Figure 3 illustrates such a comparison for a few neutron-capture elements. As it is evident from the figure, the neutron capture elements show enhanced abundances compared to normal field giants of similar metallicity.

From the location of the stars in the HR diagram $\left(\log \mathrm{T}_{\text {eff }} \mathrm{v} / \mathrm{s} \log \left(\mathrm{L} / \mathrm{L}_{\odot}\right)\right.$ plot $)$, it is clear that HD 207585 is on the subgiant branch (SGB) and the stars HD 36650 and HD 219116 are on the first ascent of giant branch (RGB). Though the star HD 207585 is classified in SIMBAD as a luminosity class II star,our analysis shows that this object is a subgiant star with log g value 3.80. The estimate of $\log$ g using the parallax method gives a value 3.90 which is consistent with the spectroscopic estimate. Our estimate is close to the literature values 4.00 (Masseron et al. 2010) and 3.50 (Smith \& Lambert 1986a). At these evolutionary stages of these stars, the s-process nucleosynthesis is not likely to happen and hence it is likely that the observed overabundance of heavy elements are extrinsic. A binary mass-transfer scenario, in which the products of s-process nucleosynthesis from a companion (now invisible white dwarf) AGB star contaminated the surface of the now observed barium star could explain this observed overabundance. According to Jorissen et al. (1998), binarity is a necessary condition to produce Ba stars, but it is not a sufficient condition.

We have also estimated the abundance ratios $[\mathrm{ls} / \mathrm{Fe}],[\mathrm{hs} / \mathrm{Fe}]$ and $[\mathrm{hs} / \mathrm{ls}]$, where ls represents light s-process elements $\mathrm{Sr}, \mathrm{Y}$ and $\mathrm{Zr}$, and hs represents heavy s-process elements $\mathrm{Ba}, \mathrm{La}, \mathrm{Ce}$ and $\mathrm{Nd}$. The 


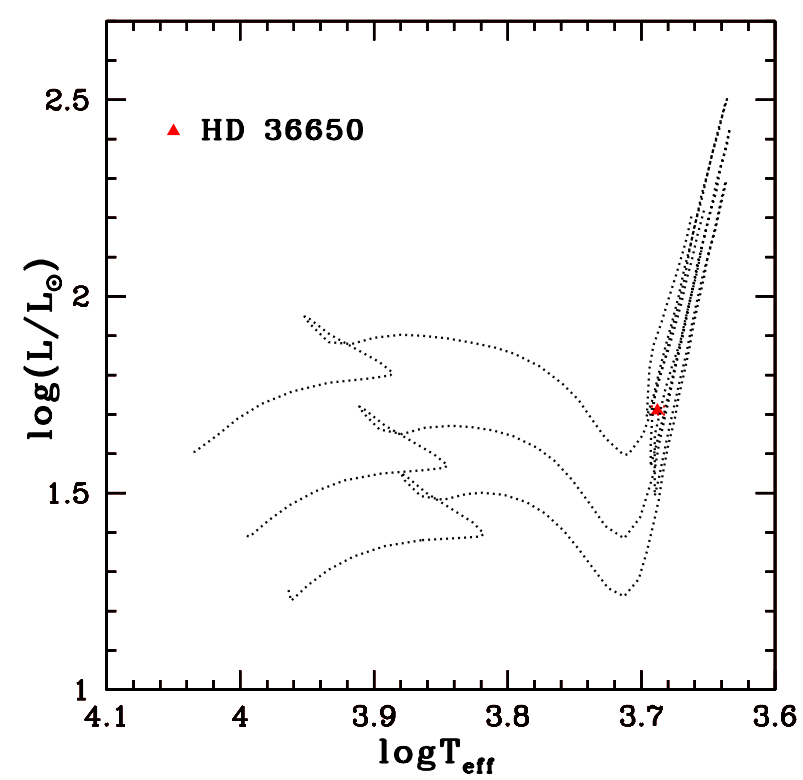

Figure 2: Location of HD 36650 in the Hertzsprung-Russell diagram. The evolutionary tracks are shown for 2.0, 2.2 and $2.5 M_{\odot}$ from bottom to top for $\mathrm{z}=0.019$.

Table 3: [hs/ls] ratios of program stars

\begin{tabular}{|l|l|l|l|l|}
\hline Star name & {$[\mathrm{Fe} / \mathrm{H}]$} & {$[\mathrm{ls} / \mathrm{Fe}]$} & {$[\mathrm{hs} / \mathrm{Fe}]$} & {$[\mathrm{hs} / \mathrm{ls}]$} \\
\hline HD 36650 & -0.02 & 0.62 & 0.84 & 0.22 \\
HD 207585 & -0.38 & 1.29 & 1.66 & 0.37 \\
HD 219116 & -0.45 & 0.71 & 1.32 & 0.61 \\
\hline
\end{tabular}

[hs/ls] values are given in Table 3. [hs/ls] is an indicator of s-process efficiency. As the metallicity decreases, the number of seed nuclei for neutron-capture decreases, and more neutrons per seed nuclei will be available. This favours the production of heavy s-process elements. As a result, the [hs/ls] ratio increases with decreasing metallicity (Clayton 1988, Wallerstein 1997, Goriely \& Mowlavi 2000, Busso et al. 2001, de Castro et al. 2016, Cristallo et al. 2011). According to the AGB models of Busso et al. (2001), at metallicities higher than solar, the light s-process elements (ls) are the dominant product over heavy s-process elements (hs). At lower metallicities, hs are the dominant products over ls. All these three stars show [hs/ls] $>0$, indicating the overabundance of Ba-peak elements over Sr-peak elements. Our estimates of [hs/ls] agree with the model calculations of Busso et al. (2001) for similar metallicities.

The ${ }^{13} \mathrm{C}(\alpha, \mathrm{n}){ }^{16} \mathrm{O}$ reaction is the major neutron source in low-mass AGB stars with initial mass $\leq 3 M_{\odot}$. The neutron density produced by this source is $\sim 10^{8}$ neutrons $/ \mathrm{cm}^{3}$ which is much lower than the density, $\sim 10^{13}$ neutrons $/ \mathrm{cm}^{3}$, produced by ${ }^{22} \mathrm{Ne}(\alpha, \mathrm{n})^{25} \mathrm{Mg}$ (Busso et al. 2001, Goriely \& Mowlavi 2000). The efficiency of the neutron source ${ }^{13} \mathrm{C}(\alpha, \mathrm{n}){ }^{16} \mathrm{O}$ is anti-correlated with metallicity (Clayton 1988, Wallerstein 1997). Hence low [hs/ls] ratios can be expected for the low mass stars at near solar metallicities considering the neutron source ${ }^{13} \mathrm{C}(\alpha, \mathrm{n}){ }^{16} \mathrm{O}$. Also, massive AGB stars with masses between 5 and $8 M_{\odot}$ can show low [hs/ls] ratios considering the neutron source ${ }^{22} \mathrm{Ne}(\alpha$, n) ${ }^{25} \mathrm{Mg}$ (Busso et al. 2001, Karakas \& Lattanzio 2014). But in massive AGB stars, Na and Mg are strongly produced as a result of ${ }^{22} \mathrm{Ne}$ burning (Bisterzo et al. 2010). But none of our stars show 
enhanced abundances of $\mathrm{Na}$ and $\mathrm{Mg}$. This may indicate the operation of the ${ }^{13} \mathrm{C}$ source in the former AGB companion, which in turn suggests a low mass for the companion.

Another indicator of neutron density and mass of AGB stars is the ratio $[\mathrm{Rb} / \mathrm{Sr}]$. Neutron density required for the production of $\mathrm{Rb}$ from $\mathrm{Kr}$ is $\sim 5 \times 10^{8}$ neutrons $/ \mathrm{cm}^{3}$ indicating the operation of the ${ }^{22} \mathrm{Ne}(\alpha, \mathrm{n}){ }^{25} \mathrm{Mg}$ reaction. In low-mass stars, $\mathrm{Sr}$ is produced instead. Thus the ratio [ $\left.\mathrm{Rb} / \mathrm{Sr}\right]$ should be positive in massive AGB stars (Karakas et al. 2012). We have searched for Rb lines in our spectra. The $\mathrm{Rb}$ I line at $7947.597 \AA$ returns a $[\mathrm{Rb} / \mathrm{Sr}]$ value -0.56 in HD 36650 . We could not determine the $\mathrm{Rb}$ abundance in other stars as no useful lines were detected in their spectra for the abundance analysis.

We have compared the neutron-capture elemental abundance ratios of the program stars with that of low-mass AGB stars from literature where the ${ }^{13} \mathrm{C}$ source is operating. The comparison is shown in Figure 4. The abundance ratios are found to match closely. The scatter in the ratio may be a consequence of different dilution factors during the mass transfer as well as the orbital parameters, metallicity and initial mass (de Castro et al. 2016).

\section{Conclusions}

We have conducted a detailed spectroscopic analysis for a sample of three barium stars. The abundance estimates are consistent with the operation of the ${ }^{13} \mathrm{C}(\alpha, \mathrm{n}){ }^{16} \mathrm{O}$ source in the former low-mass AGB companion. Detection of Rb I line at 7947.597 $\AA$ in the spectrum of HD 36650 allowed us to determine the $[\mathrm{Rb} / \mathrm{Sr}]$ ratio with a value of -0.56 . This ratio indicates the operation of ${ }^{13} \mathrm{C}(\alpha, \mathrm{n}){ }^{16} \mathrm{O}$ in the companion star. As this reaction occurs in the low-mass AGB stars, we confirm that the former companion of HD 36650 is a low-mass AGB star. Distribution of abundance patterns and [hs/ls] ratios indicate low-mass companions for the objects HD 207585 and HD 219116. While in this work we have presented the results from the analyses of three objects, a detailed study including a larger sample is in preparation.

\section{Acknowledgements}

The authors would like to acknowledge the organizers of the $2^{\text {nd }}$ BINA workshop for the local hospitality and the financial support. SJ would also like to acknowledge IIA for partial financial support. Funding from DST SERB project No. EMR/2016/005283 is gratefully acknowledged. AG would also like to acknowledge the financial support from BINA projects DST/INT/Belg/P-02 (India) and BL/11/IN07 (Belgium).

\section{References}

Abia C., Wallerstein G. 1998, MNRAS, 293, 89

Allen D. M., Barbuy B. 2006, A\&A, 454, 895

Alonso A., Arribas S., Martinez-Roger C. 1999, A\&ASS, 140, 261

Asplund M., Grevesse N., Sauval A.J. et al. 2009, ARA\&A, 47, 481

Barbuy B., Jorissen A., Rossi S. C. F. et al. 1992, A\&A, 262, 216

Bartkevicius A. 1996, Balt. Astron., 5, 217

Bisterzo S., Traglio C., Wiescher M. et al. 2010, MNRAS, 404, 1529

Bidelman W. P., Keenan P. C. 1951, ApJ, 114, 473

Busso M., Gallino R., Wasserburg G. J. 1999, ARA\&A, 37, 239

Busso M., Gallino R., Lambert D. L. et al. 2001, ApJ, 557, 802

Cayrel R., Depagne E., Spite M. et al. 2004, A\&A, 416, 1117 


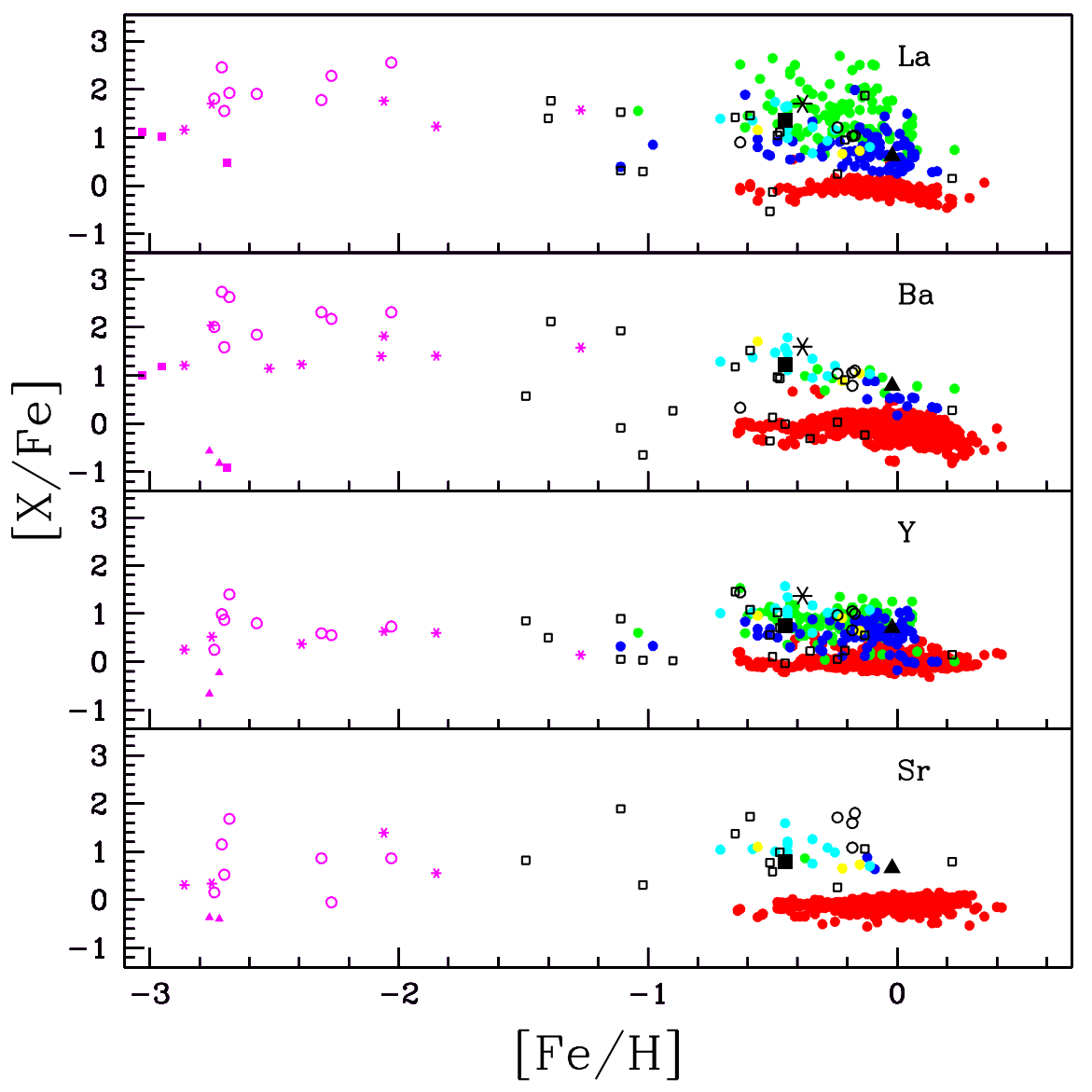

Figure 3: Abundance ratios of heavy elements $\mathrm{Sr}, \mathrm{Y}, \mathrm{Ba}, \mathrm{La}$ observed in the program stars as a function of metallicity $[\mathrm{Fe} / \mathrm{H}]$. Red circles represent normal giants from literature (Luck \& Heiter 2007). Green, blue, cyan, yellow circles represent strong Ba giants, weak Ba giants, Ba dwarfs, Ba subgiants respectively from literature (de Castro et al. 2016, Yang et al. 2016, Allen \& Barbuy 2006). Magenta stars, filled squares, open circles, filled triangles represent the CEMP-s, CEMP-r, CEMP-r/s, CEMP-no stars respectively from literature (Masseron et al. 2010). Black open squares, open circles represent $\mathrm{CH}$ giants and subgiants respectively from literature (Karinkuzhi \& Goswami 2014, 2015, Goswami et al. 2006, 2016, Sneden \& Bond 1976, Vanture 1992, Goswami \& Aoki 2010, Jonsell et al. 2006, Masseron et al. 2010). HD 36650 (solid triangle), HD 207585 (six sided cross), HD 219116 (solid square). 


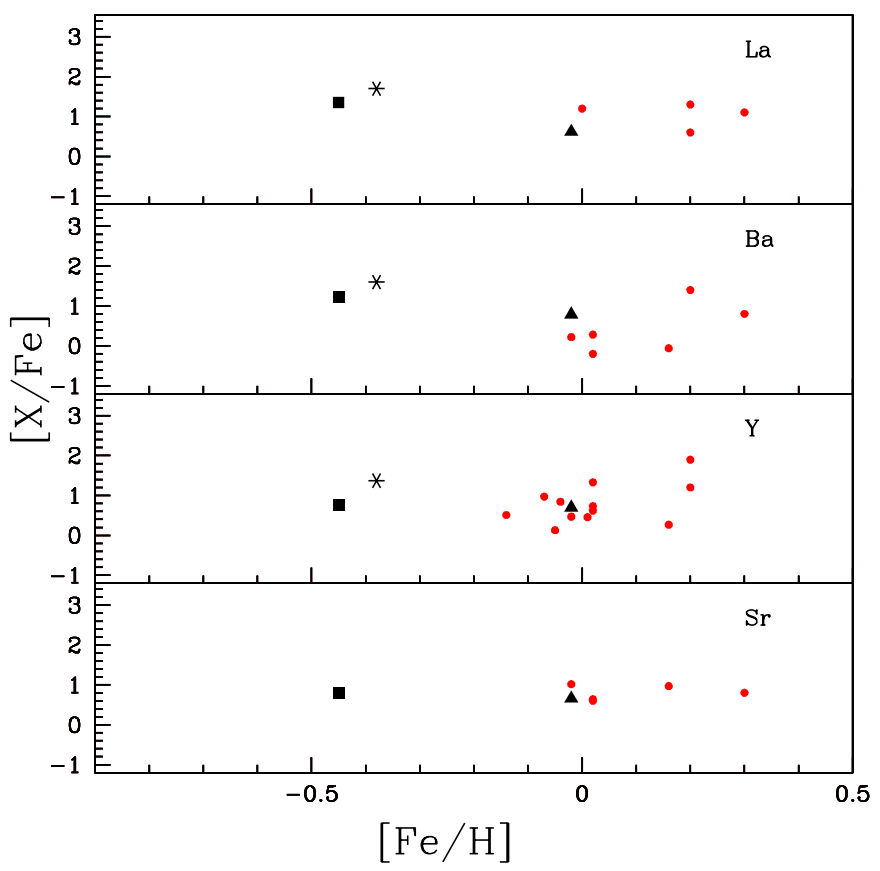

Figure 4: Comparison of abundance ratios of neutron-capture elements observed in the program stars and the AGB stars with respect to metallicity $[\mathrm{Fe} / \mathrm{H}]$. Red circles represent the AGB stars from literature (Smith \& Lambert 1985, 1986b, 1990, Abia \& Wallerstein 1998).

Chen B., Vergely J. L, Valette B. et al. 1998, A\&A, 336,137

Clayton D. D. 1988 , MNRAS, 234, 1

Cristallo S., Piersanti L., Sraniero O. et al. 2011, ApJS, 197,17

de Castro D. B., Pereira C. B., Roig F. et al. 2016, MNRAS, 459, 4299

Drake N. A., Pereira C. B. 2008, AJ, 135, 1070

Dray L. M., Tout C. A., Karakas A. I. et al. 2003, MNRAS, 338, 973

Girardi L., Bressan A., Bertelli G. et al. 2000, A\&AS, 141, 371

Goriely S., Mowlavi N. 2000, A\&A, 362, 599

Goswami A., Aoki W., Beers T. C. et al. 2006 ,MNRAS, 372, 343

Goswami A., Aoki W. 2010, MNRAS, 404, 253

Goswami A., Aoki W., Karinkuzhi D. 2016, MNRAS, 455, 402

Henry R. B. C., Edmunds M. G., Köppen J. 2000, ApJ, 541, 660

Jonsell K., Barklem P. S., Gustafsson B. et al. 2006, A\&A, 451, 651

Jorissen A., Van Eck S., Mayor M. et al. 1998, A\&A, 332, 877

Jorissen A., Boffin H. M. J., Karinkuzhi D. et al. 2019, A\&A, in press (arXiv:1904.03975)

Karakas A. I., Garcia-Hernandez D. A., Lugaro M. 2012, ApJ, 751, 8

Karakas A. I., Lattanzio J. C. 2014, PASA, 31, 30

Karinkuzhi D., Goswami A. 2014, MNRAS, 440, 1095

Karinkuzhi D., Goswami A. 2015, MNRAS, 446, 2348

Lu P. K. 1991, AJ, 101, 2229

Luck R. E., Bond H. E. 1991, ApJS, 77, 515

Luck R. E., Heiter U. 2007,AJ, 133, 2464

Masseron T., Johnson J. A., Plez B. et al. 2010, A\&A, 509, A93

McClure R. D. 1983, ApJ, 268, 264

McClure R. D. 1984, PASP, 96, 117

McClure R. D., Woodsworth W. 1990, ApJ, 352, 709

McClure R. D., Fletcher J. M., Nemec J. 1980, ApJ, 238, L35

McWilliam A. 1998, AJ, 115, 1640

Pereira C. B., Drake N. A. 2009, A\&A, 496, 791 
Prochaska J. X., McWilliam A. 2000, ApJ, 537, L57

Prochaska J. X., Naumov S. O., Carney B. W. et al. 2000, AJ, 120, 2513

Smith V. V., Lambert D. L. 1985, ApJ, 294, 326

Smith V. V., Lambert D. L. 1986a, ApJ, 303, 226

Smith V. V., Lambert D. L. 1986b, ApJ, 311, 843

Smith V. V., Lambert D. L. 1990, ApJS, 72, 387

Sneden C. 1973, Ph.D. Thesis, Univ. of Texas

Sneden C., Bond H. E. 1976, ApJ, 204,810

Udry S., Jorissen A., Mayor M. et al. 1998a, A\&AS, 131, 25

Udry S., Mayor M., Van Eck S. et al. 1998b, A\&AS, 131, 43

Vanture A. 1992, AJ, 104, 5

Wallerstein G. 1997, RvMP, 69,995

Worely C. C., Hill V. J., Sobeck J. et al. 2013, A\&A, 553, A47

Yang G. C., Liang Y. C., Spite M. et al. 2016, RAA, 16, 19 